\title{
3'-Deoxy-3'-[ $\left.{ }^{18} \mathrm{~F}\right]$ Fluorothymidine Uptake is Related to Thymidine Phosphorylase Expression in Various Experimental Tumor Models
}

${ }^{1}$ Sonja Schelhaas, ${ }^{2 *}$ Kathrin Heinzmann, ${ }^{2}$ Davina J. Honess, ${ }^{2}$ Donna-Michelle Smith, ${ }^{3}$ Heather Keen, ${ }^{4}$ Sandra Heskamp, ${ }^{5 *}$ Timothy H. Witney, ${ }^{6}$ Laurent Besret, ${ }^{7}$ Sabrina Doblas, ${ }^{2}$ John R. Griffiths, ${ }^{5}$ Eric O. Aboagye, ${ }^{1,8}$ Andreas $\mathrm{H}$. Jacobs

${ }^{1}$ European Institute for Molecular Imaging (EIMI), Westfälische Wilhelms-Universität (WWU) Münster, Münster, Germany

${ }^{2}$ Cancer Research UK Cambridge Institute, University of Cambridge, Cambridge, UK

${ }^{3}$ PHB Imaging Group, AstraZeneca, Alderley Park, Macclesfield, UK

${ }^{4}$ Radboud University Medical Centre, Department of Radiology and Nuclear Medicine, Nijmegen, The Netherlands

${ }^{5}$ Comprehensive Cancer Imaging Centre, Imperial College London, London, UK

${ }^{6}$ Sanofi Oncology, Vitry-sur-Seine, France

7 LBI, UMR1149-CRI, INSERM, Paris, France

${ }^{8}$ Department of Geriatric Medicine, Johanniter Hospital, Bonn, Germany

${ }^{*}$ current address: Comprehensive Cancer Imaging Centre, Imperial College London, London, UK

${ }^{* *}$ Current address: UCL Centre for Advanced Biomedical Imaging, University College London, London, UK

\section{Corresponding author:}

Prof. Dr. med. Andreas H. Jacobs, European Institute for Molecular Imaging (EIMI), Waldeyerstr. 15, D-48149 Münster, Phone: +492518349300, Fax:+492518349313, email: ahjacobs@uni-muenster.de

Running title: TP and [18 $\mathrm{F}] \mathrm{FLT}$ in a range of tumor models

Manuscript category: Brief article 


\section{ABSTRACT}

Purpose: We recently reported that high thymidine phosphorylase (TP) expression is accompanied by low tumor thymidine concentration and high $3^{\prime}-$ deoxy- $3^{\prime}-\left[{ }^{18} \mathrm{~F}\right]$ fluorothymidine ([18 $\left.\left.\mathrm{F}\right] \mathrm{FLT}\right)$ uptake in four untreated lung cancer xenografts. Here, we investigated whether this relationship also holds true for a broader range of tumor models.

Procedures: Lysates from $n=15$ different tumor models originating from $n=6$ institutions were tested for TP and thymidylate synthase (TS) expression using western blots. Results were correlated to $\left[{ }^{18} \mathrm{~F}\right] \mathrm{FLT}$ accumulation in the tumors as determined by positron emission tomography (PET) measurements in the different institutions and to previously published thymidine concentrations.

Results: Expression of TP correlated positively with $\left[{ }^{18} \mathrm{~F}\right] \mathrm{FLT}$ SUV $\max (\rho=0.549, \quad P<0.05)$. Furthermore, tumors with high TP levels possessed lower levels of thymidine $(\rho=-0.939, P<0.001)$.

Conclusions: In a broad range of tumors, $\left[{ }^{18} \mathrm{~F}\right] \mathrm{FLT}$ uptake as measured by PET is substantially influenced by TP expression and tumor thymidine concentrations. These data strengthen the role of TP as factor confounding $\left[{ }^{18} \mathrm{~F}\right] \mathrm{FLT}$ uptake.

Key words: $\left[{ }^{18} \mathrm{~F}\right] \mathrm{FLT}, \mathrm{PET}$, thymidine phosphorylase, oncology

\section{INTRODUCTION}

Imaging of tumor metabolism is important in characterizing the viable active tumor and monitoring response to therapy. As a thymidine analog, the positron emission tomography (PET) tracer 3'-deoxy$3^{\prime}-\left[{ }^{18} \mathrm{~F}\right]$ fluorothymidine $\left(\left[{ }^{18} \mathrm{~F}\right] \mathrm{FLT}\right)$ is a potential biomarker for imaging of tumor proliferation at baseline and in response to therapy. In analogy to thymidine, $\left[{ }^{18} \mathrm{~F}\right] \mathrm{FLT}$ is transported into cells, primarily via the human equilibrative nucleoside transporter 1 (hENT1, [1]), where it is phosphorylated by thymidine kinase 1 (TK1). As an alternative thymidine-to-DNA pathway, phosphorylated thymidine can be generated by methylation of deoxyuridine monophosphate by thymidylate synthase (TS) (Fig. 1). Even though numerous studies demonstrate a good correlation of $\left[{ }^{18} \mathrm{~F}\right] \mathrm{FLT}$ with cellular proliferation in tumors [2, 3], several reports show that factors like thymidine kinase $1[4,5]$ or thymidine [6] also affect uptake of this radiotracer. Recently, we demonstrated that $\left[{ }^{18} \mathrm{~F}\right] \mathrm{FLT}$ uptake cannot be directly 
related to proliferation, as determined by immunohistochemistry of Ki67 or 5-bromo-2'-deoxyuridine (BrdUrd) in four non-small cell lung cancer (NSCLC) xenograft models. We showed that high [ $\left.{ }^{18} \mathrm{~F}\right] \mathrm{FLT}$ accumulation was detectable in tumors with high thymidine phosphorylase (TP) expression [7]. This was in agreement with a study, demonstrating an association of TP immunohistochemical score and $\left[{ }^{18} \mathrm{~F}\right] \mathrm{FLT}$ retention in a clinical setting [8]. In our preclinical study, the presence of TP was accompanied by the corresponding enzymatic activity [7], which results in degradation of thymidine [9]. $\left[{ }^{18} \mathrm{~F}\right] \mathrm{FLT}$ is resistant to the catabolic activity of TP $[10,11]$. Hence, in tumors with high TP activity thymidine is degraded. Consequently, lower amounts of thymidine compete with $\left[{ }^{18} \mathrm{~F}\right] \mathrm{FLT}$ for cellular uptake and retention, resulting in higher $\left[{ }^{18} \mathrm{~F}\right] \mathrm{FLT}$ accumulation, as schematically depicted in Fig. 1. The relationship between the enzyme and $\left[{ }^{18} \mathrm{~F}\right] \mathrm{FLT}$ retention has already been demonstrated by infusion of TP in a preclinical model in vivo [12] and by TP inhibition in vitro [8], whereas in the latter setting, it was shown that TP inhibition does not alter TK1 or hENT1 activity.

Here, we aimed to identify whether the relationship of TP expression, thymidine levels and $\left[{ }^{18} \mathrm{~F}\right] \mathrm{FLT}$ uptake can be detected in a broad range of untreated tumors. Hence, we investigated various tumor models from different institutions with respect to their expression of thymidine metabolism proteins, and compared these data with respective $\left[{ }^{18} \mathrm{~F}\right] \mathrm{FLT}$ uptake and published thymidine concentrations.

\section{MATERIALS AND METHODS}

\section{Animal models and study design}

Data were analyzed from $n=6$ institutions. These institutions are members of the preclinical workpackage of the Innovative Medicine Initiative Joint Undertaking project QulC-ConCePT (grant agreement $\mathrm{N}^{\circ}$ 115151). The institutions are: AstraZeneca, Macclesfield, UK (AZ); CRUK Cambridge Institute, Cambridge, UK (CI); Imperial College London, London, UK (IC); Radboud University Medical Centre, Nijmegen, The Netherlands (Radboudumc); Westfälische Wilhelms-Universität Münster, Münster, Germany (WWU); INSERM, Paris, France (INSERM); and Sanofi Oncology, Vitry-sur-Seine, France (Sanofi), performing the PET imaging for the INSERM tumors.

All animal experiments were carried out by the members of the consortium in accordance with the EU Directive 2010/63/EU and the NCRI Guidelines for the welfare and use of animals in cancer research [13]. The tumor models were chosen in each institute as appropriate for their future evaluation of response to specific therapies. Here, only untreated animals were used to assess $\left[{ }^{18} \mathrm{~F}\right] \mathrm{FLT}$ 
confounding factors at baseline. Study designs differed for the different institutions, and cohorts used for western blot analysis, $\left[{ }^{18} \mathrm{~F}\right] \mathrm{FLT}$ PET scans, and thymidine analysis partly overlap, so that in total $n=209$ animals were included in this study. All tumors were grown as subcutaneous xenografts or allografts, except for the CC531 liver metastasis model from Radboudumc. Details about the models are listed in Table 1.

\section{Western blot analysis}

Immediately after excision, tumors were frozen in liquid nitrogen, stored at $-80^{\circ} \mathrm{C}$, and shipped to WWU on dry ice for analysis of thymidine metabolism proteins. Tumor tissue was homogenized in RIPA buffer (Cell Signaling) by a micro-dismembrator and $20 \mu \mathrm{g}$ per sample were subjected to western blot analysis. The PVDF membrane was incubated overnight at $4{ }^{\circ} \mathrm{C}$ with specific primary antibodies. Afterwards the membrane was probed with appropriate peroxidase-coupled secondary antibodies for 1 hour at room temperature. See Supplementary Table 1 for antibody details. Protein bands were visualized with Pierce ECL Plus Western Blotting Substrate (Pierce Biotechnology).

Band intensities were quantified by ImageJ (National Institutes of Health), using the "gel analyzer" function, and normalized to the actin loading control. To normalize for unequal exposures of different blots, all band intensities were expressed relative to a control sample (an H1975 lysate) that was loaded on all gels.

\section{$\left[{ }^{18} \mathrm{~F}\right] \mathrm{FLT}$ PET imaging of small animals}

The scanning procedures of each institution are listed in Table 2. The animals were not fasted. For quantification, standardized uptake values (SUV $\max$ and $S U V_{\text {mean }}$ ) were determined.

\section{Statistics}

Data are expressed as mean \pm standard deviation. Numerical values, as well as number of samples analyzed, are depicted in Supplementary Table 2. Spearman correlations were calculated (SigmaPlot 13.0 ) and $P$-values $<0.05$ were considered statistically significant. 


\section{RESULTS}

Tumor lysates from untreated rodents were analyzed for TP and TS expression. The representative western blot as well as the quantification revealed large differences between tumors (Fig. 2). We related the TP and TS expression levels to $\left[{ }^{18} \mathrm{~F}\right] \mathrm{FLT}$ uptake in the respective tumors and detected a statistically significant correlation between $\left[{ }^{18} \mathrm{~F}\right] \mathrm{FLT}$ SUV $\max$ and TP levels $(\rho=0.549, P<0.05$, Fig. 3). Also SUV mean was significantly correlated with TP levels $(\rho=0.582, P<0.05$, Supplementary Fig. 1). No relation between TS and $\left[{ }^{18} \mathrm{~F}\right] \mathrm{FLT}$ or TS and TP was observed.

When relating TP expression to respective previously published tumor thymidine concentrations [14] (confirmation of assay robustness presented in Supplementary Fig. 2), we observed a significant correlation ( $\rho=-0.939, P<0.001$, Fig. 4A). Moreover, tumor thymidine was inversely correlated with $\left[{ }^{18} \mathrm{~F}\right] \mathrm{FLT}$ SUV $\max$ (Fig. 4B) and $\left[{ }^{18} \mathrm{~F}\right] \mathrm{FLT}$ SUV $V_{\text {mean }}$ (Supplementary Fig. 3).

\section{DISCUSSION}

Defining the molecular and cellular determinants of $\left[{ }^{18} \mathrm{~F}\right] \mathrm{FLT}$ uptake and retention in tumors helps to determine the potential value of this tracer in clinical oncology. Here, we demonstrate that in various tumor models $\left[{ }^{18} \mathrm{~F}\right] \mathrm{FLT}$ accumulation is influenced by the presence of the enzyme TP, which accounts for degradation of endogenous thymidine. We observed a statistically significant correlation of TP expression with $\left[{ }^{18} \mathrm{~F}\right] \mathrm{FLT}$ uptake (Fig. 3B and Supplementary Fig. 1). Moreover, we were able to compare the TP data of ten tumor models with tumor thymidine data published previously [14]. As expected, TP expression negatively correlated with thymidine levels and these thymidine concentrations negatively correlated with $\left[{ }^{18} \mathrm{~F}\right] \mathrm{FLT}$ (Fig. 4). The thymidine data strengthen the TP results presented here, as they provide a link between protein expression and activity. Of note, the data analyzed by Heinzmann et al. [14] only partly overlap with the data presented here, explaining slight differences in the results.

For some tumor models, plasma thymidine levels were available. This parameter would be more easily accessible in the clinical setting. Although these plasma thymidine values weakly correlated with tumor thymidine values in this subset of models, no direct relation of this parameter with TP expression or $\left[{ }^{18} \mathrm{~F}\right] \mathrm{FLT}$ uptake could be detected (Supplementary Fig. 4). However, as the sample size for some of the models is low, these data should be treated with caution. 
In our setup, immunohistochemistry, $\left[{ }^{18} \mathrm{~F}\right] \mathrm{FLT}$ PET measurements and the thymidine assay were only partly performed using the same animals. When looking at overlapping datasets on a per-tumor-basis, available from a total of $n=54$ tumors, a significant correlation of $\left[{ }^{18} \mathrm{~F}\right] \mathrm{FLT}$ with TP and thymidine, as well as TP with thymidine can also be detected (Supplementary Fig. 5), strengthening the conclusion, that these factors are related.

In addition to the single institution studies published so far, covering only a limited spectrum of models $[7,8,12]$, our data provide evidence that the relationship of $\left[{ }^{18} \mathrm{~F}\right] \mathrm{FLT}$, thymidine and TP can be detected in a wider range of tumors in a multi-center setting. This reinforces the role of TP as a factor affecting $\left[{ }^{18} \mathrm{~F}\right] \mathrm{FLT}$ uptake. This is of importance for the implementation of this tracer as imaging biomarker for tumor therapy follow up, potentially improving therapy in clinical cancer research.

Ex vivo analysis of TP expression might potentially help in determining the utility of [ $\left.{ }^{18} \mathrm{~F}\right] \mathrm{FLT}$ PET for a specific tumor model. Some high proliferating tumors might have low $\left[{ }^{18} \mathrm{~F}\right] \mathrm{FLT}$ uptake at baseline due to low TP expression and are not good candidates for $\left[{ }^{18} \mathrm{~F}\right] \mathrm{FLT}$ response studies. Accordingly, some of the tumors analyzed here, showing high TP expression and high $\left[{ }^{18} \mathrm{~F}\right] \mathrm{FLT}$ uptake, could be successfully utilized for preclinical monitoring of tumor therapies $[15,16]$.

As the moderate correlation coefficient between TP and $\left[{ }^{18} \mathrm{~F}\right] \mathrm{FLT}$ SUV $\max$ suggests $(\rho=0.549$, $P<0.05)$, other factors also influence $\left[{ }^{18} \mathrm{~F}\right] \mathrm{FLT}$ uptake. It is well recognized that nucleoside transporters and TK1 are involved in $\left[{ }^{18} \mathrm{~F}\right] \mathrm{FLT}$ accumulation. Consequently, care must be taken when employing therapies targeting $\left[{ }^{18} \mathrm{~F}\right] \mathrm{FLT}$ modulating factors, as shown already for TS-inhibitors [3]. Our study provides evidence that the same might hold true for agents modulating TP or thymidine concentrations. For instance, taxol and oxaliplatin can increase TP levels $[17,18]$. Hence, TP and thymidine should be considered when employing $\left[{ }^{18} \mathrm{~F}\right] \mathrm{FLT}$ PET in combination with a novel treatment approach. If a treatment does not affect an $\left[{ }^{18} \mathrm{~F}\right] \mathrm{FLT}$ confounding component, changes in $\left[{ }^{18} \mathrm{~F}\right] \mathrm{FLT}$ uptake can still reflect treatment response, as described in a range of systematic reviews [3,19]. In our study, $\left[{ }^{18} \mathrm{~F}\right] \mathrm{FLT}$ uptake was determined in different institutions with different scanners and in different rodent models. Therefore, it is even more striking that a statistically significant correlation of $\left[{ }^{18} \mathrm{~F}\right] \mathrm{FLT}$ uptake with TP could be observed. These variables also differ in the clinical situation. Hence, we speculate that this relation could also be detected in clinical specimens. Lee et al. have demonstrated an association of $\left[{ }^{18} \mathrm{~F}\right] \mathrm{FLT}$ and TP immunohistochemistry score in a cohort of 58 nonsmall cell lung cancer patients. However, no thymidine analysis was performed [8]. It is known that plasma thymidine levels in humans are much lower than in rodents $[6,20]$ and that TP levels vary 
between clinical tumor specimens [21]. Whether this variation is related to thymidine and [ $\left.{ }^{18} \mathrm{~F}\right] \mathrm{FLT}$ in the respective tumors remains to be determined.

One limitation of the present study is the fact that protein expression, as determined here, is not necessarily directly related to enzymatic activity. Furthermore, quantification of expression levels via western blot densitometry is only semi-quantitative [22]. Of note, TP western blot results are in line with TP levels determined by immunohistochemistry, as we reported previously [7]. This is of importance, as immunohistochemistry is a more clinically accessible method.

\section{CONCLUSION}

$\left[{ }^{18} \mathrm{~F}\right] \mathrm{FLT}$ accumulation in tumors can be influenced by competition with endogenous thymidine, which might be controlled by TP. Hence, our data provide further evidence that TP is a major factor influencing $\left[{ }^{18} \mathrm{~F}\right] \mathrm{FLT}$ uptake, which should be taken into account when employing this radiotracer in tumor therapy follow up studies.

\section{ACKNOWLEDGEMENTS}

The research leading to these results has received support from the Innovative Medicines Initiative Joint Undertaking (www.imi.europa.eu) under grant agreement number 115151, resources of which are composed of financial contribution from the European Union's Seventh Framework Programme (FP7/2007-2013) and EFPIA companies' in kind contribution. This work was also supported by the Deutsche Forschungsgemeinschaft (DFG), Cells-in-Motion Cluster of Excellence (EXC1003 - CiM), University of Münster, and the Interdisciplinary Centre for Clinical Research (IZKF, core unit PIX), Münster, Germany.

\section{CONCFLICT OF INTEREST}

The authors declare that they have no conflict of interest.

\section{ETHICAL APPROVAL}

All applicable institutional and national guidelines for the care and use of animals were followed. 


\section{ELECTRONIC SUPPLEMENTARY MATERIAL}

Supplementary Fig. 1 TP expression correlated to $\left[{ }^{18} \mathrm{~F}\right] \mathrm{FLT}$ uptake in the tumor models investigated

Supplementary Fig. 2 Inter-experiment reproducibility of the thymidine assay

Supplementary Fig. 3 Relation of $\left[{ }^{18} \mathrm{~F}\right] \mathrm{FLT}$ SUV mean to tumor thymidine levels

Supplementary Fig. 4 Plasma thymidine concentrations are not correlated with TP expression or $\left[{ }^{18} \mathrm{~F}\right] \mathrm{FLT}$ uptake in tumors

Supplementary Fig. 5 When analyzing data on a per-tumor-basis, the relation of $\left[{ }^{18} \mathrm{~F}\right] \mathrm{FLT}$, TP and thymidine can also be detected

Supplementary Table 1 Antibodies used for western blot analysis

Supplementary Table 2 Numerical values of data presented in this manuscript, as well as number of samples analyzed (in brackets)

\section{REFERENCES}

1. Paproski RJ, Ng AML, Yao SYM, Graham K, Young JD, Cass CE (2008) The role of human nucleoside transporters in uptake of 3'-deoxy-3'-fluorothymidine. Mol Pharmacol 74:1372-1380

2. Chalkidou A, Landau DB, Odell EW, Cornelius VR, O'Doherty MJ, Marsden PK (2012) Correlation between Ki-67 immunohistochemistry and 18F-fluorothymidine uptake in patients with cancer: A systematic review and meta-analysis. Eur J Cancer 48:3499-3513

3. Schelhaas S, Heinzmann K, Bollineni VR, Kramer GM, Liu Y, Waterton JC, Aboagye EO, Shields AF, Soloviev D, Jacobs AH (2017) Preclinical Applications of 3'-Deoxy-3'[18F]Fluorothymidine in Oncology - A Systematic Review. Theranostics 7:40-50

4. McKinley ET, Ayers GD, Smith RA, Saleh SA, Zhao P, Washington MK, Coffey RJ, Manning HC (2013) Limits of [18F]-FLT PET as a biomarker of proliferation in oncology. PLoS One 8:e58938

5. Barthel H, Perumal M, Latigo J, He Q, Brady F, Luthra SK, Price PM, Aboagye EO (2005) The uptake of 3'-deoxy-3'-[18F]fluorothymidine into L5178Y tumours in vivo is dependent on thymidine kinase 1 protein levels. Eur J Nucl Med Mol Imaging 32:257-263

6. Zhang CC, Yan Z, Li W, et al (2012) [(18)F]FLT-PET imaging does not always "light up" 
proliferating tumor cells. Clin Cancer Res 18:1303-1312

7. Schelhaas S, Wachsmuth L, Viel T, et al (2014) Variability of proliferation and diffusion in different lung cancer models as measured by 3'-deoxy-3'-18F-fluorothymidine PET and diffusion-weighted MR imaging. J Nucl Med 55:983-988

8. Lee SJ, Yeo JS, Lee HJ, Lee EJ, Kim SY, Jang SJ, Lee JJ, Ryu J-S, Moon DH (2014) Thymidine phosphorylase influences [(18)F]fluorothymidine uptake in cancer cells and patients with non-small cell lung cancer. Eur J Nucl Med Mol Imaging 41:1327-1335

9. Liekens S, Bronckaers A, Pérez-Pérez M-J, Balzarini J (2007) Targeting platelet-derived endothelial cell growth factor/thymidine phosphorylase for cancer therapy. Biochem Pharmacol 74:1555-1567

10. Barthel H, Aboagye E, Price P (2003) Reply. Cancer Res 63:8560-8560

11. Toyohara J, Fujibayashi $Y$ (2003) Trends in nucleoside tracers for PET imaging of cell proliferation. Nucl Med Biol 30:681-5

12. Van Waarde A, Cobben DCP, Suurmeijer AJH, Maas B, Vaalburg W, De Vries EFJ, Jager PL, Hoekstra HJ, Elsinga PH (2004) Selectivity of 18F-FLT and 18F-FDG for differentiating tumor from inflammation in a rodent model. J Nucl Med 45:695-700

13. Workman P, Aboagye EO, Balkwill F, et al (2010) Guidelines for the welfare and use of animals in cancer research. Br J Cancer 102:1555-1577

14. Heinzmann K, Honess DJ, Lewis DY, et al (2016) The relationship between endogenous thymidine concentrations and [(18)F]FLT uptake in a range of preclinical tumour models. EJNMMI Res 6:63

15. Schelhaas S, Held A, Wachsmuth L, Hermann S, Honess DJ, Heinzmann K, Smith D-M, Griffiths JR, Faber C, Jacobs AH (2016) Gemcitabine Mechanism of Action Confounds Early Assessment of Treatment Response by 3'-Deoxy-3'-[18F]Fluorothymidine in Preclinical Models of Lung Cancer. Cancer Res 76:7096-7105

16. Leyton J, Smith G, Lees M, Perumal M, Nguyen Q, Aigbirhio FI, Golovko O, He Q, Workman P, Aboagye EO (2008) Noninvasive imaging of cell proliferation following mitogenic extracellular kinase inhibition by PD0325901. Mol Cancer Ther 7:3112-3121 
17. Sawada N, Ishikawa T, Fukase Y, Nishida M, Yoshikubo T, Ishitsuka H (1998) Induction of thymidine phosphorylase activity and enhancement of capecitabine efficacy by taxol/taxotere in human cancer xenografts. Clin Cancer Res 4:1013-9

18. Cassidy J, Tabernero J, Twelves C, et al (2004) XELOX (capecitabine plus oxaliplatin): active first-line therapy for patients with metastatic colorectal cancer. J Clin Oncol 22:2084-91

19. Bollineni VR, Kramer GM, Jansma EP, Liu Y, Oyen WJG (2016) A systematic review on [18F]FLT-PET uptake as a measure of treatment response in cancer patients. Eur J Cancer $55: 81-97$

20. Li KM, Clarke SJ, Rivory LP (2003) Quantitation of plasma thymidine by high-performance liquid chromatography_atmospheric pressure chemical ionization mass spectrometry and its application to pharmacodynamic studies in cancer patients. Anal Chim Acta 486:51-61

21. Han J-Y, Hong EK, Lee SY, Yoon SM, Lee DH, Lee JS (2005) Thymidine phosphorylase expression in tumour cells and tumour response to capecitabine plus docetaxel chemotherapy in non-small cell lung cancer. J Clin Pathol 58:650-4

22. Heidebrecht F, Heidebrecht A, Schulz I, Behrens SE, Bader A (2009) Improved semiquantitative Western blot technique with increased quantification range. $\mathrm{J}$ Immunol Methods 345:40-48 


\section{FIGURES}

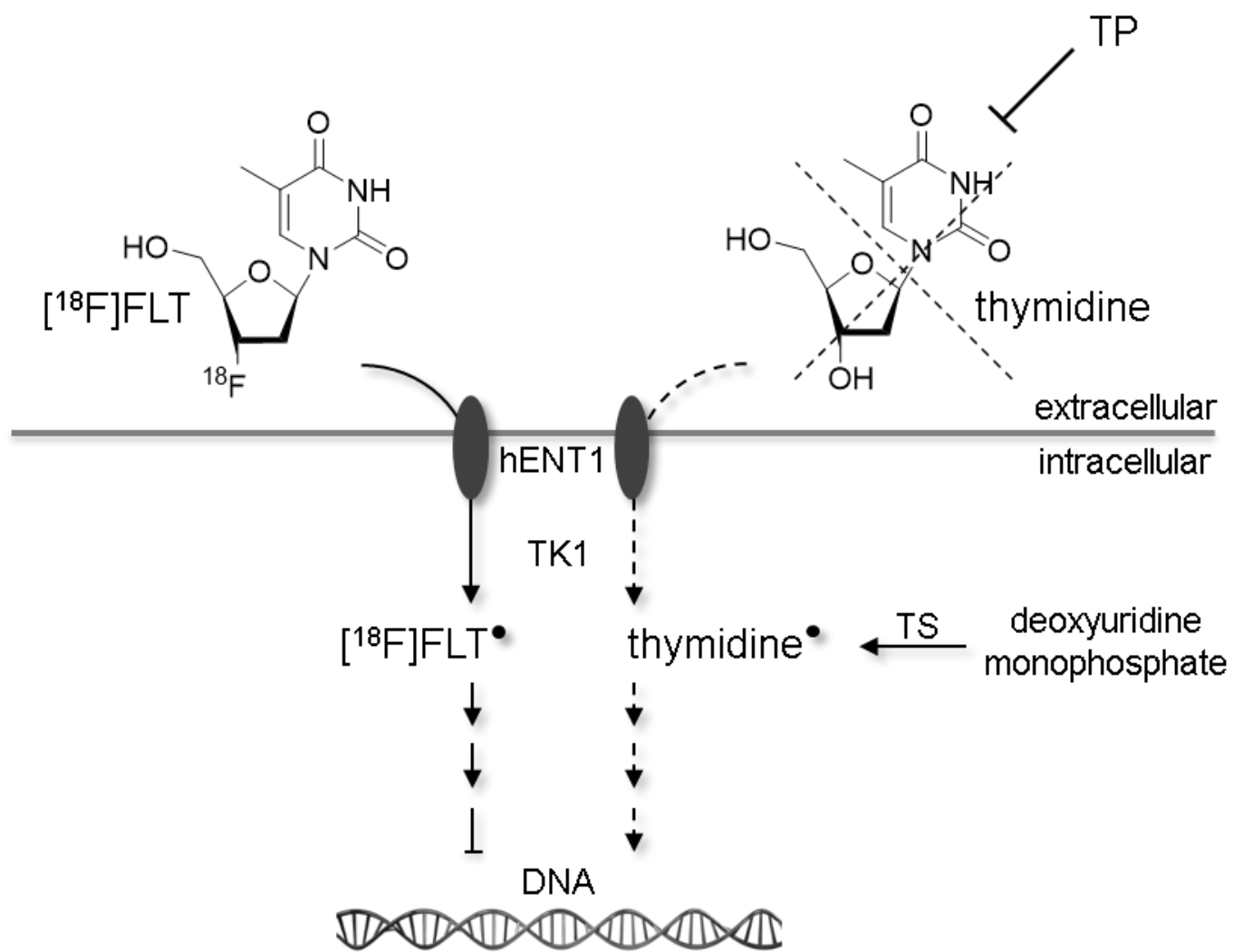

Fig. 1 Schematic illustration of the competition between uptake of $\left[{ }^{18} \mathrm{~F}\right] \mathrm{FLT}$ and thymidine and the influence of TP. When thymidine is degraded by TP, as indicated by dotted lines, less of this nucleoside competes with $\left[{ }^{18} \mathrm{~F}\right] \mathrm{FLT}$ for uptake into cells, or the phosphorylation (indicated by a black dot) via TK1. hENT1, as the major transporter, and TS, as the key enzyme of the alternative thymidine-to-DNA pathway, are also shown. 
a

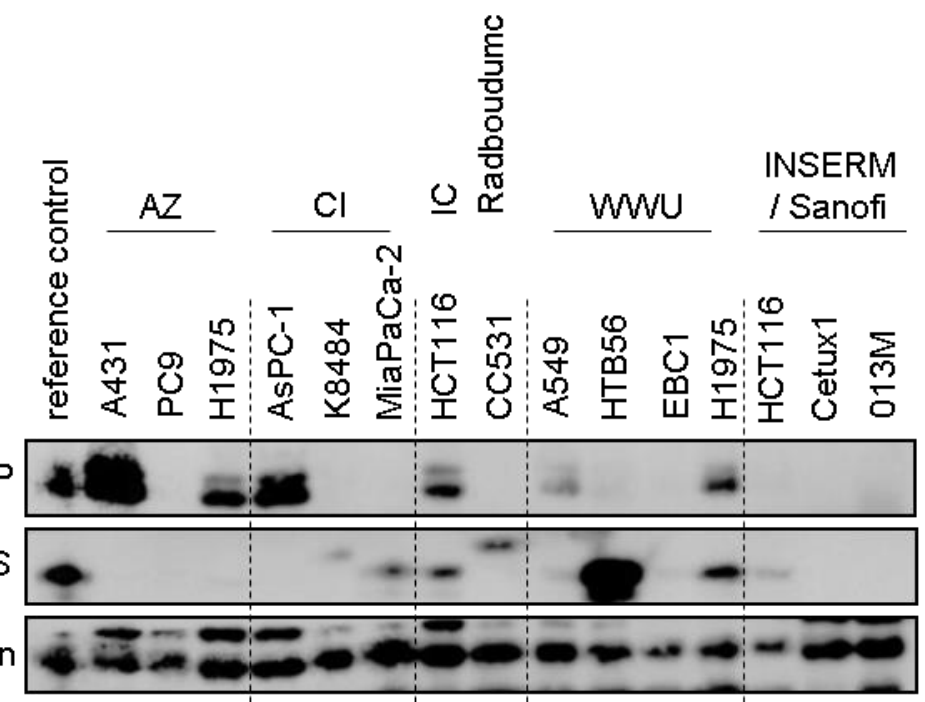

b
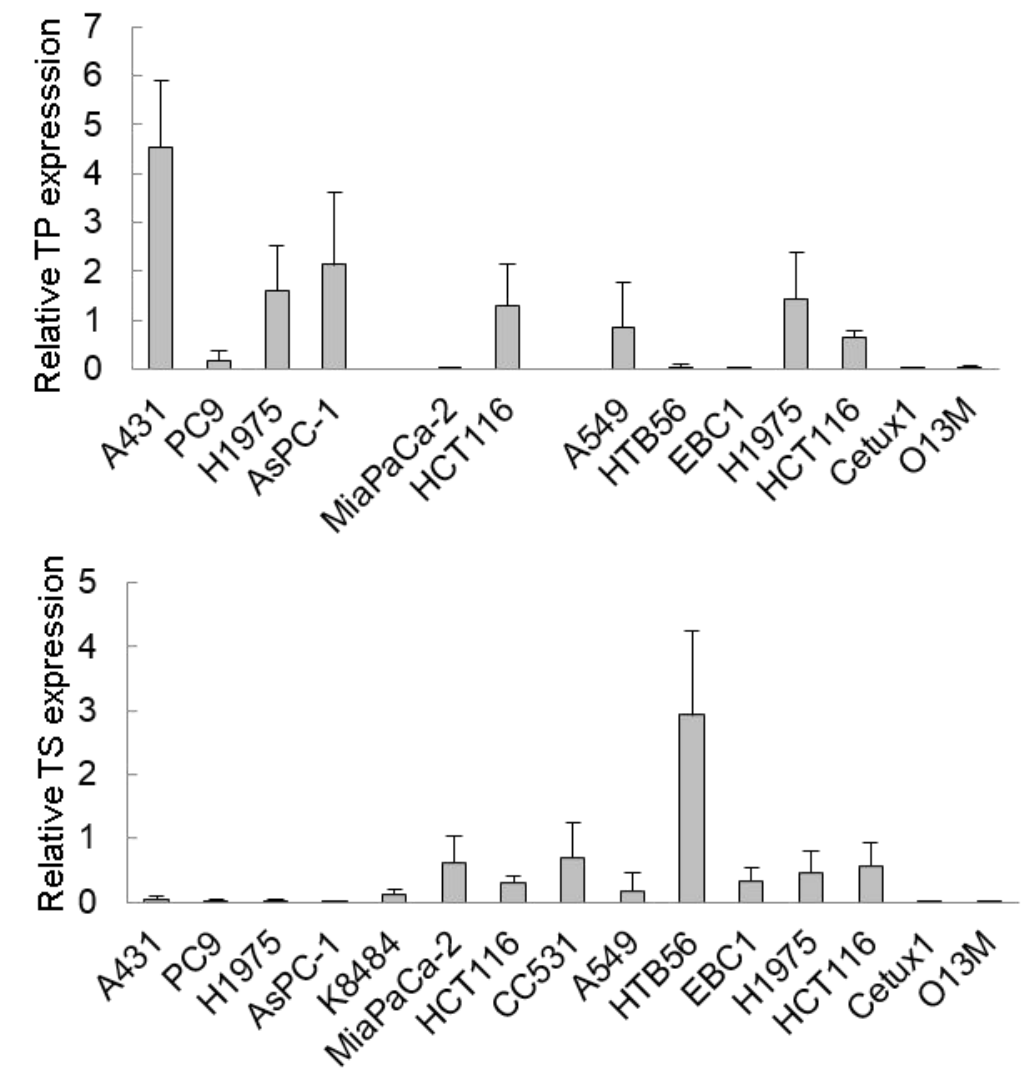

Fig. 2 Western blot analysis revealed varying amounts of thymidine metabolism proteins in different tumor models. Actin was used as loading control (a). Quantification of TP (b) and TS (c) expression was performed via densitometric analysis. The TS band of the rodent K8484 and CC351 tumors is slightly shifted, which can probably be attributed to species-specific differences in the protein sequence. The TP antibody used is not optimized for rodent samples. Hence, these samples were excluded from any correlations. 
a

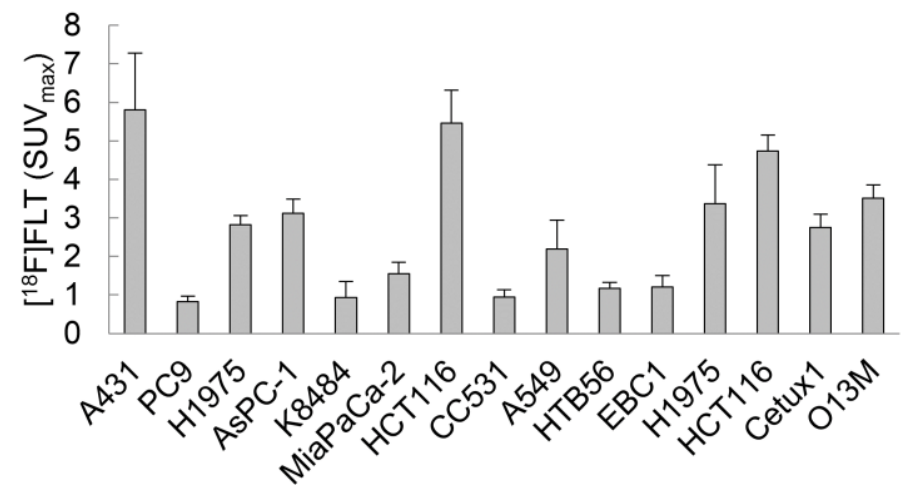

b

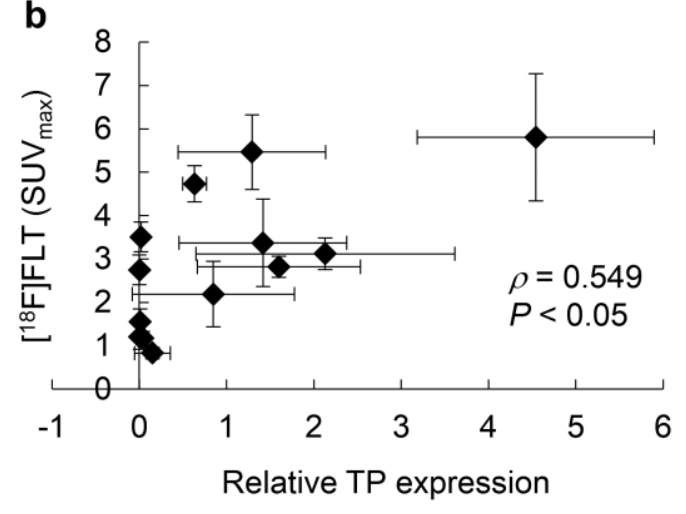

Fig. 3 TP expression was significantly correlated to $\left[{ }^{18} \mathrm{~F}\right] \mathrm{FLT}$ uptake in the tumor models investigated. $\left[{ }^{18} \mathrm{~F}\right] \mathrm{FLT}$ SUV $\max$ was determined by PET measurements in the different institutions (a). Correlation was assessed by the Spearman method (b).
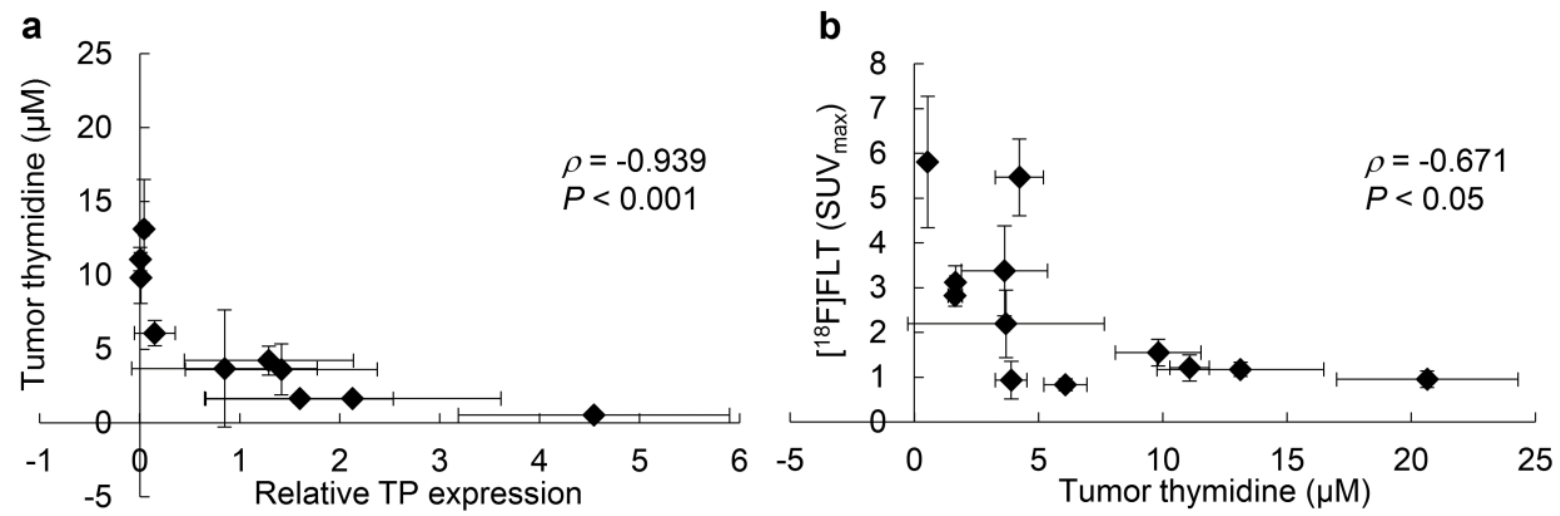

Fig. 4 Correlation of TP, thymidine and $\left[{ }^{18} \mathrm{~F}\right] \mathrm{FLT}$. We related our findings of western blot and PET analyses to tumor thymidine levels. Thymidine contents were inversely correlated to TP levels (a) and $\left[{ }^{18} \mathrm{~F}\right] \mathrm{FLT}$ uptake was inversely correlated with thymidine concentrations (b). 


\section{TABLES}

Table 1. Information on investigated tumor models.

\begin{tabular}{|c|c|c|c|c|}
\hline Institution & Cell line & Tumor origin & Model type & Host (supplier) \\
\hline \multirow[t]{3}{*}{$\mathrm{AZ}$} & A431 & human epidermis & Xenograft & AP ONU mouse (AZ) \\
\hline & PC9 & human lung & Xenograft & AP CB17 SCID mouse (AZ) \\
\hline & H1975 & human lung & Xenograft & AP ONU mouse (AZ) \\
\hline \multirow[t]{3}{*}{$\mathrm{Cl}$} & AsPC-1 & human pancreas & Xenograft & CB17 SCID mouse (CR) \\
\hline & K8484 & murine pancreas & Syngeneic allograft & $\begin{array}{l}\text { PC mouse (p53R172H; Pdx1- } \\
\text { Cre) (CRUK Cl) }\end{array}$ \\
\hline & MiaPaCa-2 & human pancreas & Xenograft & CB17 SCID mouse (CR) \\
\hline IC & HCT116 & human colorectum & Xenograft & BALB/c nu mouse (CR) \\
\hline Radboudumc & CC531 & rat colorectum & $\begin{array}{l}\text { Syngeneic liver } \\
\text { metastasis }\end{array}$ & Wag/Rij rats (CR) \\
\hline \multirow[t]{4}{*}{ WWU } & A549 & human lung & Xenograft & NMRI nu (Janvier) \\
\hline & HTB56 & human lung & Xenograft & NMRI nu (Janvier) \\
\hline & EBC1 & human lung & Xenograft & NMRI nu (Janvier) \\
\hline & H1975 & human lung & Xenograft & NMRI nu (Janvier) \\
\hline \multirow{3}{*}{$\begin{array}{l}\text { INSERM / } \\
\text { Sanofi }\end{array}$} & HCT116 & human colorectum & Xenograft & CB17 SCID (CR) \\
\hline & $\begin{array}{l}\text { CR-IC-O13M- } \\
\text { Cetux1 }\end{array}$ & $\begin{array}{l}\text { human colon - } \\
\text { hepatic metastasis }\end{array}$ & Xenograft & CB17 SCID (CR) \\
\hline & CR-IC-O13M & human colon & Xenograft & CB17 SCID (CR) \\
\hline
\end{tabular}

$\mathrm{CR}=$ Charles River 
Table 2. PET imaging and analysis parameters

\begin{tabular}{|c|c|c|c|c|c|c|c|}
\hline Institution & PET scanner & $\begin{array}{l}\text { imaging time } \\
\text { (min) }\end{array}$ & $\begin{array}{l}\text { injected dose } \\
(\mathrm{MBq})\end{array}$ & ROls defined on & $\begin{array}{l}\text { reconstruction } \\
\text { method }\end{array}$ & voxel size (mm) & $\begin{array}{l}\text { matrix size } \\
\text { (pixel) }\end{array}$ \\
\hline$A Z$ & Inveon (Siemens) & $50-60$ & $\sim 10$ & PET & 2D-FBP & $0.77 \times 0.77 \times 0.80$ & $128 \times 128 \times 159$ \\
\hline $\mathrm{Cl}$ & NanoPET/CT (Mediso) & $60-90$ & 8.3 & CT & 3D OSEM & $0.4 \times 0.4 \times 0.4$ & $255 \times 255 \times 236$ \\
\hline IC & Inveon (Siemens) & $50-60$ & $\sim 3.7$ & CT & 3D-OSEM/MAP & $0.8 \times 0.8 \times 0.8$ & $128 \times 128 \times 159$ \\
\hline Radboudumc & Inveon (Siemens) & $60-75$ & $10-12$ & PET & 3D-OSEM & $0.43 \times 0.43 \times 0.8$ & $256 \times 256 \times 159$ \\
\hline WWU & $\begin{array}{l}\text { quadHIDAC (Oxford } \\
\text { Positron Systems) }\end{array}$ & $70-90$ & $\sim 10$ & CT & 3D-OPL-EM & $0.4 \times 0.4 \times 0.4$ & $150 \times 150 \times 300$ \\
\hline $\begin{array}{l}\text { Sanofi/ } \\
\text { INSERM }\end{array}$ & Inveon (Siemens) & $75-90$ & $\sim 7$ & PET & 3D-OSEM & $0.30 \times 0.30 \times 0.796$ & $256 \times 256 \times 97$ \\
\hline
\end{tabular}

